\title{
MULTIPLE DENTIGEROUS CYSTS IN A CHILD: A CASE REPORT AND
RADIOGRAPHIC FOLLOW-UP
}

\section{ABSTRACT}

The aim of this study is to describe a case of dentigerous cysts of a young patient successfully treated as well as clinical and radiographic follow-up. A 10-year-old female patient was referred to the Buccomaxillofacial Surgery and Traumatology Service of the Regional University Hospital of Campos Gerais. The patient's panoramic radiograph revealed a well-defined radiolucent unilocular area associated to the crown of the second permanent right lower molar, which was impacted. In addition, the permanent upper canines were enclosed, suggesting a dentigerous cyst. A conservative treatment was determined, through marsupialization of the lesions. The histopathology examination confirmed the clinical diagnosis of dentigerous cysts. Longitudinal clinical and radiographic monitoring was essential to certify the regression of the lesion, as well as bone neoformation, thus promoting the eruption and maintenance of the affected permanent teeth.

Keywords: Dentigerous cyst, panoramic radiography, diagnosis.

\author{
D Bruna Carolyne Siefert de Oliveira \\ Amanda Regina Fischborn ${ }^{2}$ \\ (D) $*$ Caique Mariano Pedroso ${ }^{1}$ \\ (D) Jéssica Daniela Andreis ${ }^{2}$ \\ (iD) Camila Maggi Maia Silveira ${ }^{3}$ \\ (D) Luiz Felipe Manosso Guzzoni ${ }^{1}$ \\ (D) Marcelo Carlos Boroluzzi ${ }^{4}$ \\ (D) Gilson Cesar Nobre Franco ${ }^{4}$
}

ORCID IDs of the authors:
$\begin{array}{ll}\text { B.C.S.O. } & 0000-0001-6247-2941 \\ \text { A.R.F. } & 0000-0002-7470-484 X \\ \text { C.M.P. } & 0000-0002-7504-7597 \\ \text { J.D.A. } & 0000-0002-4435-4850 \\ \text { C.M.M.S. } & 0000-0001-6434-5532 \\ \text { L.F.M.G } & 0000-0002-7032-4516 \\ \text { M.C.B. } & 0000-0003-2756-5047 \\ \text { G.C.N.F. } & 0000-0001-7082-7837\end{array}$

1 State University of Ponta Grossa, Ponta Grossa - PR, Brazil.

${ }^{2}$ MSc in Integrated Clinical (Etiology and Diagnosis of Oral Diseases), State University of Ponta Grossa, Ponta Grossa - PR, Brazil.

3 Integrated Clinical, Department of Dentistry, State University of Ponta Grossa, Ponta Grossa -PR, Brazil.

${ }^{4}$ Diagnosis and surgery, Department of Dentistry, State University of Ponta Grossa, Ponta Grossa -PR, Brazil.

How to Cite: de Oliveira BCS, Fischborn AR, Pedroso CM, Andreis JD, Silveira CMM, Guzzoni LFM, Boroluzzi MC, Franco GCN. Multiple Dentigerous Cysts in a Child: A Case Report and Radiographic Follow-Up. Cumhuriyet Dent J 2020;23:2;136-141.

*Corresponding Author:

State University of Ponta Grossa, Ponta Grossa-PR, Brazil.

Email: caiquemp@yahoo.com.br 


\section{INTRODUCTION}

Dentigerous cysts are responsible for approximately $24 \%$ of true cysts and they are the most frequent after root cysts. ${ }^{1,2,3}$ The occurrence of these cysts is higher in men at the posterior region of the mandible, followed by the maxilla front region. The teeth usually involved are the lower third molar, upper canine, third upper molar, lower pre-molar, supernumerary tooth or ectopic tooth, respectively. ${ }^{1,4,5}$ These are usually asymptomatic lesions, randomly investigated, when a permanent tooth eruption is delayed, or as an imagiological finding during radiographic examination. It appears as a radiolucent unilocular area, with a well-defined corticated margin, around the crown of an impacted tooth. ${ }^{4,6,7}$ Histologically, these lesions present a thin stratified epithelium, which is sometimes bilaminar along with keratinizing metaplasia. ${ }^{6,8}$ Many of the odontogenic cysts share clinical and radiographic aspects, thus, the diagnosis must be based on both radiographic and histopathological findings., ${ }^{4,6}$ Clinical management consists of enucleation or marsupialization, the latter being a more conservative approach. This method consists in joining the cyst cover to the oral mucosa, which allows the spontaneous eruption of the dental element when there is enough space. ${ }^{5,9,10,11}$

Success in the treatment of dentigerous cyst depends on the correct choice of treatment, as well as on the performance of clinical and radiographic monitoring, thus preventing recurrence. In this context, the aim of this study is to describe a case of a dentigerous cyst in a young patient, taking into account the success of the treatment and the importance of long-term radiographic follow-up.

\section{CASE REPORT}

A 10-year-old female patient was referred by her orthodontist to the service of Buccomaxillofacial Surgery and Traumatology Service of the Regional University Hospital of Campos Gerais. The radiographic image revealed a well-defined unilocular radiolucency associated to the crown of the permanent lower right second molar impacted and displaced to the lower border of the mandible, as well as the presence of a radiolucent area associated with the upper canine teeth, suggesting a dentigerous cyst. (Figure 1).

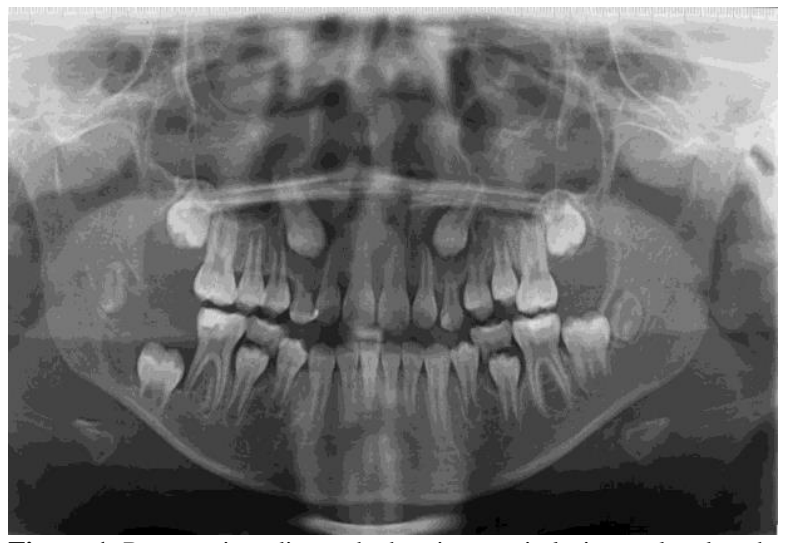

Figure 1. Panoramic radiograph showing cystic lesions related to the crown of the permanent lower right second molar and upper canines.

The clinical examination did not reveal the presence of local infection, cortical bone expansion or facial asymmetry, and the patient did not report any associated symptoms. After obtaining the free and informed consent form from her guardian, the patient was submitted to complete blood count examination, prothrombin activity time (PAT), activated partial thromboplastin time (aPTT), creatinine, urea and fasting blood glucose tests. In addition, a computerized tomography was requested to assess complementary features of the lesion, like its size and effect on adjacent structures to the lesion. The blood test results were normal, and the Computerized Tomography evidenced imaging characteristics compatible with the provisional diagnosis. (Figure 2. A-D).

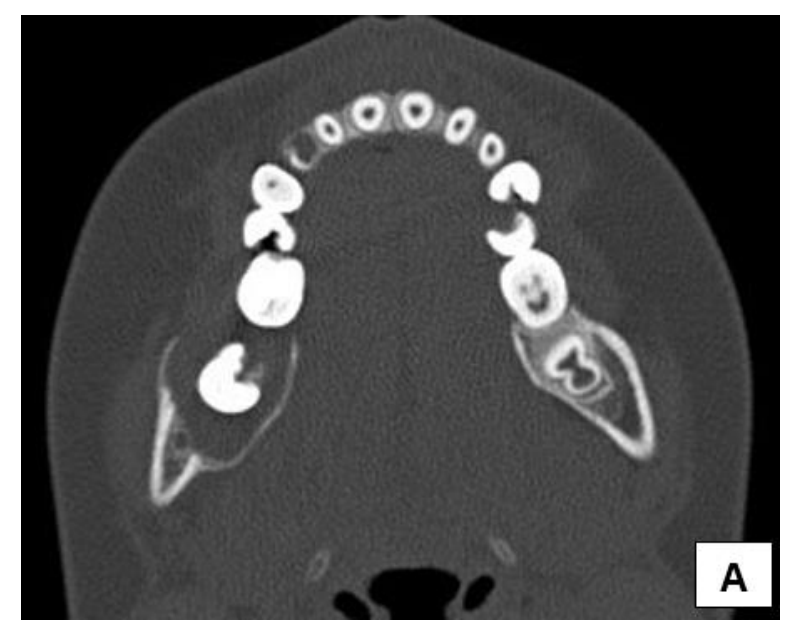




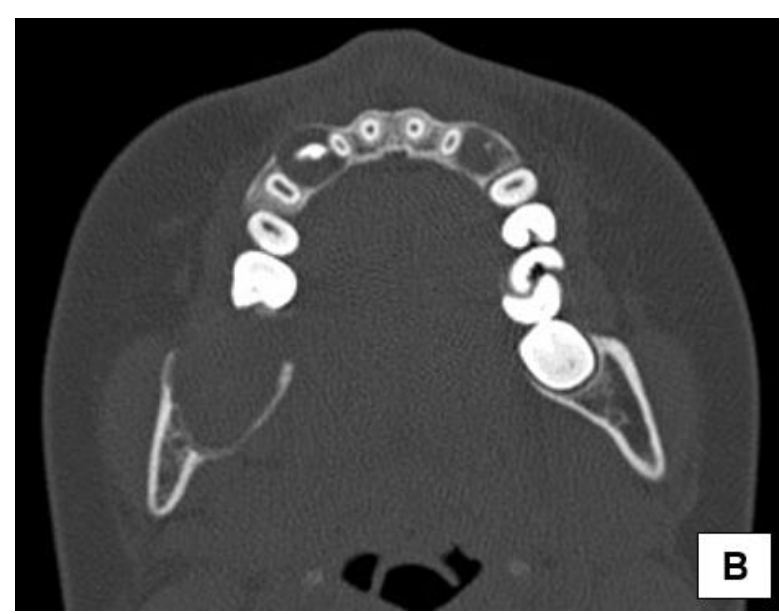

Figure 2. A-B. Computerized tomography of the face with axial cut evidencing a well-defined image of the permanent lower right second molar affected.

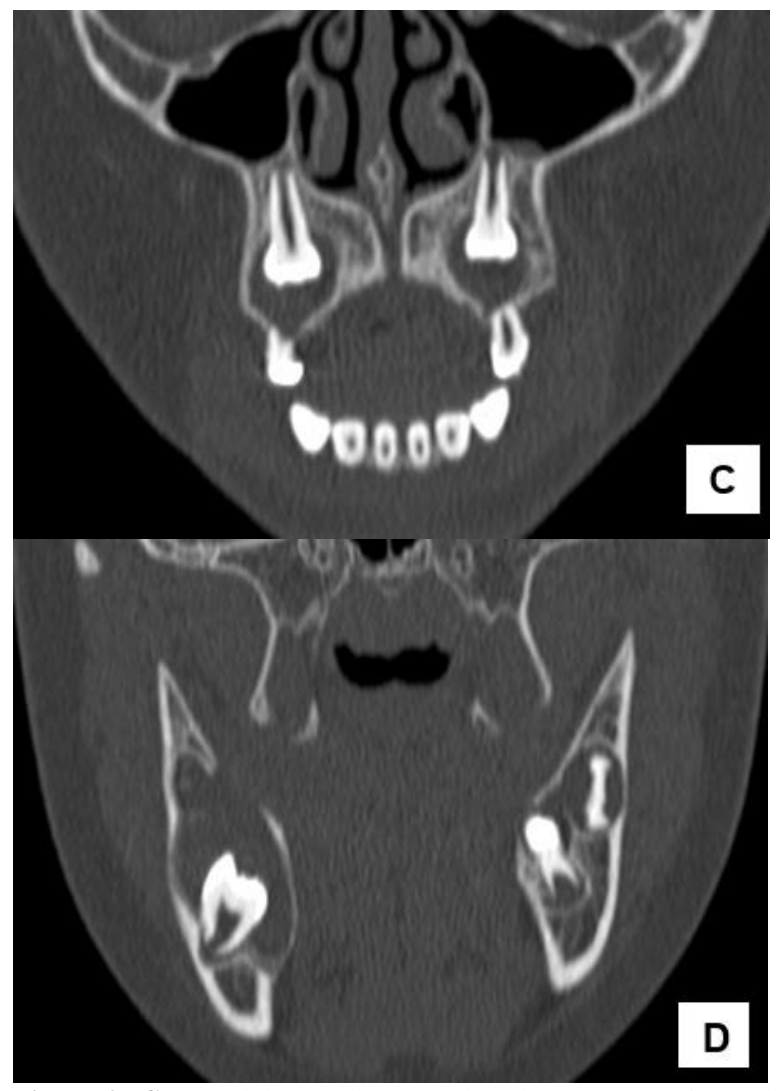

Figure 2. C-D. Axial cuts demonstrating the presence of a welldefined image of the permanent upper canines and lower right molar affected, respectively.

A conservative treatment was determined, through marsupialization of the lesions. The surgery was carried out at the hospital under general anaesthesia. During the surgery, an elliptical incision posterior to the first lower right molar was performed for the marsupialization of the cystic lesion in the adjacent tooth. The deciduous upper canines were extracted for the marsupialization of the cystic lesions of the permanent upper canines. A small sample of the material was collected and sent for histopathological analysis. After the surgery, an antibiotic therapy was prescribed consisting of amoxicillin oral suspension ( $250 \mathrm{mg} / 8$ hours) for 7 days and analgesia using dipyrone oral solution for 5 days. The patient was instructed regarding her oral hygiene, and rinsing with a $0.12 \%$ chlorhexidine solution.

The histopathological specimen showed features which were suggestive of dentigerous cysts (Figure 3).

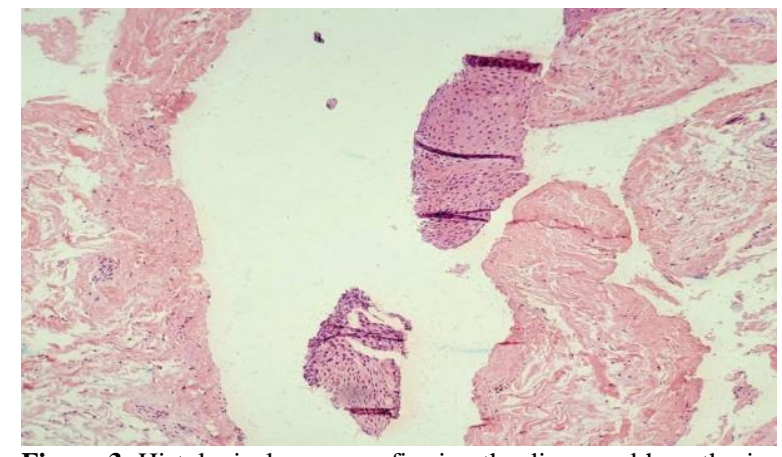

Figure 3. Histological exam confirming the diagnosed hypothesis of a typical dentigerous cyst, covered with a non-keratinized stratified epithelium. Dysplastic alterations were not observed. (Coloration H\&E, 40x).

The patient returned periodically for follow-up examinations. Suitable maintenance of the surgical site was observed and no complications were found. In the third week after the surgery, the sutures were removed. After 10 weeks, it was possible to observe clinically the cusp of the lower right second molar in an active eruption process. Within 26 weeks of surgical follow-up, the radiographic examination showed bone neoformation in the regions previously occupied by the cystic lesions and a proper tooth eruption process. In addition, the presence of a radiolucent unilocular area in the region of the right mandibular ramus was observed, however of a smaller diameter, with not well-defined borders, which might have resulted from the cystic decompression. (Figure 4. A-B).

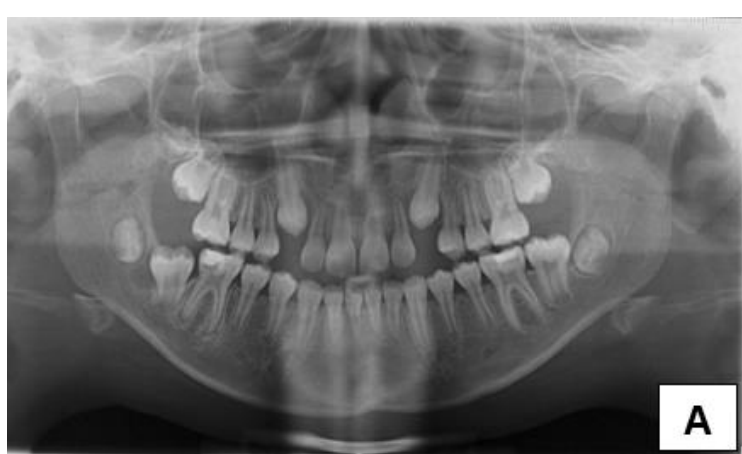




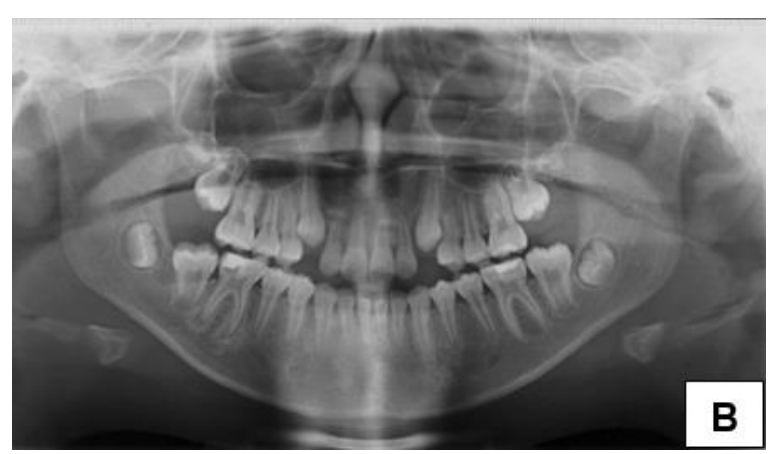

Figure 4. A-B. Radiographic follow-up after 10 weeks (A) and 26 weeks (B) post-operation, bone neo-formation was observed and a suitable eruption process of the teeth involved along with a radiolucent area on the right mandibular ramus.

After a one-year follow-up period, with radiographic evaluation, the lower right second molar was observed to be in eruption and infraocclusion process. The radiolucent area in the mandibular ramus did not show any evidence of evolution (Figure 4. C).

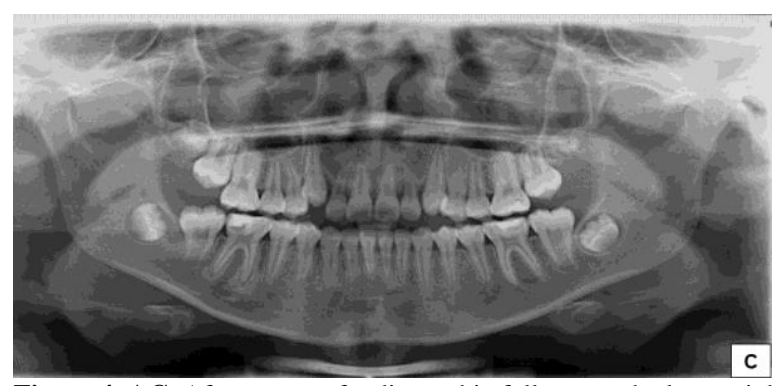

Figure 4. AC. After a year of radiographic follow-up, the lower right second molar showed infra-occlusion and a persistent radiolucent area on the mandibular ramus, without evolution.

The patient and her guardian were instructed about the importance of the radiographic analysis every six months for a suitable follow-up.

\section{DISCUSSION}

This report demonstrates a dentigerous cyst in a young patient with classic radiographic features. This odontogenic cyst is considered a common jaw lesion, and is usually found in routine radiographs at the end of the second and third decades of life. ${ }^{5,9,12}$ In this case report, the lesions showed an unusual presentation since they had an early diagnosis. According to previous studies by Ferreres et $a .^{13}$, Gendeviline et $a l^{8}$ and Langaroodi et al. ${ }^{14}$, the main teeth involved are third molars, in patients ranging in age from 15 to 34 years old. Contrasting with findings described in the literature, in the present clinical case, the involvement of lower right second molar and the maxillary canines was observed in a patient in the first decade of life.
The dentigerous cyst radiographic image revealed a well-defined unilocular radiolucy, which must be differentiated from other lesions that might present the same radiographic aspect such as odontogenic keratocyst, ameloblastoma and ameloblastic fibroma. ${ }^{12,15,16}$ Although the radiographic examination provides important information, histopathological examination is fundamental for a definite confirmatory diagnosis. ${ }^{16}$ The histopathological diagnosis criteria for dentigerous cysts include the presence of a cystic cavity with non-keratinized epithelium and a fibrous conjunctive tissue wall, ${ }^{13}$ which was observed in this case through the histopathological analysis of the sample obtained in during the surgical procedure.

Dentigerous cyst treatment options include the complete enucleation or marsupialisation, the latter being a more conservative surgical alternative. 5,9 Cases of treatment with marsupialization emphasize the relevance of choosing a less traumatic therapeutic method and preventing young patients from being exposed to unnecessary surgical procedures. In this context, this type of treatment is based on the fact that marsupialization tends to decrease the intraosseous pressure, in which the impacted tooth is able to perform its own eruption without the need for more aggressive interventions. ${ }^{17}$ Some authors use enucleation as the first choice of treatment in cases of small size cysts, as well as marsupialization previously performed followed by enucleation. ${ }^{8}$ However, the literature is not univocal regarding the best approach. In this sense, consideration should be given to the size and location, patient's age, cyst closeness to vital structures, tooth position in relation to the cyst and the degree of inclination and root formation. 15,17 Thus, the marsupialization was the treatment of choice in this case, since it is more conservative, allowing the maintenance of the permanent teeth involved. ${ }^{10}$ Although this surgical method proves to be effective, there is a controversy, in which cell debris left in situ can proliferate and consequently turn into benign or malignant odontogenic tumors. ${ }^{17}$ In our case, there 
was no recurrence of the lesion and the proposed treatment proved to be effective.

The use of general anaesthesia (GA) was the option chosen for the surgical treatment, since it is an efficient approach enabling the whole procedure to be carried out in a single session. ${ }^{18,19}$ Treatment under GA is indicated in children's treatment for several reasons such as to reduce long surgical procedures, and with extremely noncooperative patients, who might be anxious or non-cooperative, among others. ${ }^{18,19}$ The treatment can be carried out at the hospital, outpatient surgery center or at the dentist's office and presents low incidence of adverse reactions when carried out safely and efficiently by qualified professionals following the pre-established protocols and guidelines. ${ }^{20}$ In this case, the use of GA to treat patients with dentigerous cyst proved to be an effective alternative, without complications, as also occurred in the clinical case reported by Bozkurt et al. ${ }^{21}$

Ghandour et al. ${ }^{12}$ reported the use of marsupialization in a 13-year-old female patient, in which the panoramic radiograph revealed the absence of any radiolucent lesion and wellsucceeded eruption of the tooth involved. ArjonaAmo et al. ${ }^{9}$ carried out marsupialization on a young patient for the treatment of dentigerous cysts that impacted the lower first molars and after three months both teeth erupted. Therefore, marsupialization as a conservative method, can be the first option for the treatment of dentigerous cysts in young patients, thus preserving and favoring the spontaneous eruption of developing permanent teeth.

\section{CONCLUSIONS}

Dentigerous cyst management is determined by several factors, such as patient's age, cyst size, location, closeness to vital structures, among others. Marsupialization can be the first option to treat dentigerous cysts in young patients, since it is a more conservative approach that allows the eruption and maintenance of the permanent teeth involved. The importance of choosing the standardized clinical operating protocols for treatment success is highlighted. Likewise, the longitudinal radiographic follow-up is considered extremely important to the success of the treatment.

\section{ACKNOWLEDGMENTS}

None

\section{CONFLICTS OF INTEREST STATEMENT}

No conflicts of interest to disclose

\section{REFERENCES}

1. AlKhudair B, AlKhatib A, AlAzzeh G, AlMomen A. Bilateral dentigerous cysts and ectopic teeth in the maxillary sinuses: A case report and literature review. Int J Surg Case Rep 2019;55:117-120.

2. Reyes JMV, Bermúdez JAE, Ruisánchez YEG. Dentigerous Cysts: Case Report. J Adv Oral Res 2016;7(1):41-45.

3. Moturi K, Kaila V. Management of Non-syndromic Multiple Impacted Teeth with Dentigerous Cysts: A Case Report. Cureus 2018;10(9):1-7.

4. De Souza LB, Gordón-Núñez MA, Nonaka CFW, De Medeiros MC, Torres TF, Emiliano GBG. Odontogenic cysts: Demographic profile in a Brazilian population over a 38-year period. Med Oral Patol Oral Cir Bucal 2010;15(4):1-8.

5. Tayşi M, Özden C, Çankaya AB, Yıldırım S, Bilgiç

L. Conservative Approach To a Large Dentigerous Cyst in an 11 Year Old Patient. J Istanbul Univ Fac Dent 2016;50(3):51-56.

6. Allison JR, Garlington G. The value of cone beam computed tomography in the management of dentigerous cysts-a review and case report. Dent Update 201;44(3):182-188.

7. Terauchi M, Akiya S, Kumagai J, Ohyama Y, Yamaguchi S. An analysis of dentigerous cysts developed around a mandibular third molar by panoramic radiographs. Dent J 2019;7(1):1-9.

8. Gendviliene I, Legrand P, Nicolielo LFP, Sinha D, Spaey Y, Politis C, et al. Conservative management of large mandibular dentigerous cysts with a novel approach for follow up: Two case reports. Stomatologija 2017;19(1):24-32.

9. Arjona-Amo M, Serrera-Figallo MA, HernándezGuisado JM, Gutiérrez-Pérez JL, Torres-Lagares D. Conservative management of dentigerous cysts in 
children. J Clin Exp Dent 2015;7(5):671-674.

10. Contar CMM, Thomé CA, Pompermayer A, Sarot JR, Vinagre RO, Machado MÂN. Marsupialization of Dentigerous Cyst: Report of a Case. J Maxillofac Oral Surg 2015;14(S1):4-6.

11. Kamasaki Y, Sasaki Y, Fujiwara T. Management of an extensive dentigerous cyst in a 12-year-old boy. Pediatr Dent J 2009;19(2):234-239.

12. Ghandour L, Bahmad HF, Bou-Assi S. Conservative Treatment of Dentigerous Cyst by Marsupialization in a Young Female Patient: A Case Report and Review of the Literature. Case Rep Dent 2018;2018:1-6.

13. Borrás-Ferreres J, Sánchez-Torres A, AguirreUrizar JM, Gay-Escoda C. Dentigerous cyst with parietal and intracystic calcifications: A case report and literature review. J Clin Exp Dent 2018;10(3):296-299.

14. Langaroodi AJ, Zarch SHH, Rahpeyma A, Khaki $\mathrm{N}$, Esmaeilzade A, Ebrahimnejad H. A brief radiographic report from two common odontogenic cysts in jaws with follicular radiolucent appearance. Cumhuriyet Dent J 2017; 20(2): 106-112.

15. Rastogi K, Agarwal P, Chitlangia P. Mandibular Dentigerous Cyst in a 10-Year-Old Child. Int J Clin Pediatr Dent 2016;9(3):281-4.
16. Khandeparker RV, Khandeparker PV, Virginkar A, Savant K. Bilateral Maxillary Dentigerous Cysts in a Nonsyndromic Child: A Rare Presentation and Review of the Literature. Case Rep Dent 2018

17. Şahin O. Conservative management of a dentigerous cyst associated with eruption of teeth in a 7-year-old girl: a case report. J Korean Assoc Oral Maxillofac Surg 2017;43(1):S1.

18. Jankauskiene B, Virtanen JI, Kubilius R, Narbutaite J. Treatment under dental general anesthesia among children younger than 6 years in Lithuania. Med 2013;49(9):403-8.

19. Savanheimo N, Sundberg SA, Virtanen JI, Vehkalahti MM. Dental care and treatments provided under general anaesthesia in the Helsinki Public Dental Service. BMC Oral Health 2012;12(1):1-8.

20. Campbell RL, Shetty NS, Shetty KS, Pope HL, Campbell JR. Pediatric dental surgery under general anesthesia: Uncooperative children. Anesth Prog 2018;65(4):225-30.

21. Bozkurt P, Altindag A, Ilhan E, Erdem E. Kissing molars: reports of three cases involving supernumerary tooth, dentigerous cyst and fibro-osseous lesion. Cumhuriyet Dent J 2017; 20(2):72-76. 